\title{
White spot syndrome virus (WSSV) PCR-positive Artemia cysts yield PCR-negative nauplii that fail to transmit WSSV when fed to shrimp postlarvae
}

\author{
Yun-Shiang Chang ${ }^{1}$, Chu-Fang Lo ${ }^{1, *}$, Shao-En Peng ${ }^{1}$, Kuan-Fu Liu ${ }^{2}$, \\ Chung-Hsiung Wang ${ }^{3}$, Guang-Hsiung Kou ${ }^{1, *}$ \\ ${ }^{1}$ Department of Zoology, National Taiwan University, Taipei, Taiwan, ROC \\ ${ }^{2}$ Tung Kang Marine Laboratory, Taiwan Fisheries Research Institute, Tung Kang, Ping Tung, Taiwan, ROC \\ ${ }^{3}$ Department of Entomology, National Taiwan University, Taipei, Taiwan, ROC
}

\begin{abstract}
Positive results were obtained with nested white spot syndrome virus (WSSV) diagnostic PCR performed on 5 commercial brands of dry-packed Artemia cysts using several WSSV genomic sequence-specific primers. In 2 brands, PCR and nucleotide sequence analysis found $\mathrm{C} \rightarrow \mathrm{T}$ and $\mathrm{C} \rightarrow \mathrm{G}$ point mutations in the pms $146 \mathrm{WSSV}$ amplicon, but in all 5 brands, the nucleotide sequences that were successfully amplified by the $r r 1, r r 2$ and $t k$-tmk gene-specific primer sets were identical to those of Penaeus monodon WSSV. However, despite the inarguable presence of WSSV or WSSV-like template DNA, we were unable to detect WSSV by PCR in hatched nauplii derived from PCR-positive cysts or in P. monodon postlarvae fed Artemia nauplii hatched from such cysts. Most simply, these results suggested that the cysts were externally contaminated with WSSV or WSSV-like template material that was removed during hatching and washing of the nauplii. Given the small sequence variations found, it may also have been a variety of WSSV non-infectious for P. monodon or Artemia and derived from other crustaceans or arthropods in the Artemia environment. However, we could not establish this conclusively and a small possibility remained that the PCR template in these tests was derived from WSSV template present internally in the cysts and derived from infected Artemia adults. However small, this possibility must be vigorously tested, given the impact that a positive outcome could have on the shrimp industry.
\end{abstract}

KEY WORDS: White spot syndrome virus - Brine shrimp - Artemia · Penaeus monodon · WSSV diagnostic PCR $\cdot$ Amplified restriction fragment length polymorphism

\section{INTRODUCTION}

White spot syndrome virus (WSSV) is the extremely virulent causative agent of white spot syndrome, an acute, contagious disease of shrimp. It was first reported from an outbreak in cultured penaeid that occurred in NE Asia in 1993 (Nakano et al. 1994, Takahashi et al. 1994). White spot syndrome causes high mortality, and it continues to seriously affect most of the commercially cultured marine shrimp species, not

*Corresponding authors. E-mail: gracelow@ccms.ntu.edu.tw or ghkou@ccms.ntu.edu.tw just in Taiwan and Asia but globally (Chou et al. 1995, Flegel 1997, Spann \& Lester 1997).

Because WSSV has such a wide host range that extends not only to several shrimp species but also to many other crustaceans (Lo et al. 1996b), we questioned whether it would be capable of infecting or contaminating the brine shrimp Artemia (Crustacea, Anostraca). This crustacean is used worldwide for feeding fish and crustaceans (Bardach et al. 1972, Sorgeloos \& Persoone 1975, Sorgeloos 1980, Kim et al. 1996, Naessens et al. 1997). For example, Artemia nauplii are routinely used in penaeid shrimp larviculture from mysis through to postlarval (PL) stages (Cook 
\& Murphy 1969, Beard et al. 1977, Emmerson 1984). Thus, if infected or contaminated with WSSV, Artemia would constitute a serious threat to the shrimp industry. WSSV has not hitherto been positively identified either in Artemia or in Artemia cysts (sometimes mistakenly called brine shrimp 'eggs'). However, it has been speculated (Austin \& Allen 1982, Overstreet et al. 1988, Mortensen et al. 1993) that Artemia can act as a reservoir or mechanical vector for bacterial pathogens such as Bacillus, Erwinia, Micrococcus, Staphylococcus and Vibrio spp. as well as the infectious pancreatic necrosis virus (Austin \& Allen 1982, Mortensen et al. 1993). In addition, cytoplasmic, rod-shaped, virus-like particles were reported in the shell gland of Artemia ovaries as long ago as 1980 (Criel 1980) and Artemia has been used as a mechanical vector in an infectivity bioassay of Baculovirus penaei (Overstreet et al. 1988). This information suggests that Artemia has the potential to be a vector for pathogens including viruses.

In view of the great importance of Artemia in shrimp culture, we used various PCR primer pairs to screen Artemia cysts from several different sources for the presence of WSSV. We also used PCR tests to screen nauplii hatched from WSSV PCR-positive cysts and to examine Penaeus monodon experimentally fed with Artemia nauplii derived from WSSV PCR-positive cysts.

\section{MATERIALS AND METHODS}

Overall strategy for PCR tests. Five commercial brands of Artemia cysts were subjected to 2-step WSSV diagnostic PCR (Lo et al. 1996a, Lo \& Kou 1998) using either the primer set pms 146F1/R1-F2/R2 (Lo et al. 1996a) or the primer set IQ2000 (Farming IntelliGene Tech, Taipei, Taiwan). Both sets target the WSSV genomic fragment pms 146 generated by pms 146F1/ R1-F2/R2 but the IQ2000 set yields a smaller amplicon. With positive cyst samples, several other nested primer sets also designed from pms 146 were subsequently used to amplify fragments for sequencing. Sequences and amplified restriction fragment length polymorphism (ARFLP) results were then compared. In addition, 4 further primer sets were used to test for amplification of sequence-specific WSSV gene fragments derived from the coding regions of 3 recently identified WSSV genes. These were WSSV rr1 and rr2 (van Hulten et al. 2000, Tsai et al. 2000a), and the WSSV tktmk gene (Tsai et al. 2000b), which encodes a novel chimeric polypeptide of cellular-type thymidine and thymidylate kinase.

Template DNA preparation for 2-step PCR. A DNA extraction kit (Farming IntelliGene Tech) consisting of 'DTAB Solution', 'CTAB Solution' and 'Dissolving Solution' was used to prepare template DNA from pooled $100 \mathrm{mg}$ samples of Artemia cysts, Artemia nauplii, Penaeus monodon at the mysis stage and $P$. monodon $\mathrm{PL}_{15}$ with their heads removed. The DNA extraction protocol followed the instruction manual provided with the kit, and the final DNA pellet was resuspended in $200 \mu \mathrm{l}$ double-distilled water. For 2-step WSSV diagnostic PCR, the DNA concentration

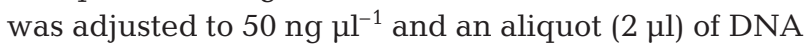
solution was used for each reaction. Positive control templates were prepared from the abdominal muscle (100 mg) of a seriously infected (1-step PCR positive; Lo \& Kou 1998, Lo et al. 1998) cultured P. monodon collected from southern Taiwan in 1994 ( $P$. monodon WSSV 1994 Taiwan isolate; Lo et al. 1996a).

Two-step WSSV diagnostic PCR. The IQ2000 primer set and all the pms 146 primer sets used here were derived from the nucleotide sequence of a cloned WSSV Sall $1461 \mathrm{bp}$ (pms 146) fragment (Lo et al. 1996a). The nucleotide sequence of the pms 146 fragment has been deposited in GenBank (accession number U50923). The pms 146F1/R1-F2/R2 nested primer set (i.e. the outer primer pair pms 146F1/R1 and the inner primer pair pms 146F2/R2) has already been described (Lo et al. 1996a). The other primer set (IQ2000) was provided as part of a commercially available WSSV detection kit (Farming IntelliGene Tech; Lo et al. 1998). It consists of an outer primer pair, 146Fup/WNFdown, and an inner primer pair, Nestup/ Nestdown2 (Farming IntelliGene Tech). The first and second steps of amplification with the IQ2000 primer set were expected to amplify DNA fragments of $910 \mathrm{bp}$ and $296 \mathrm{bp}$, respectively.

The quality of the DNA extracted from the tested Artemia and Penaeus monodon specimens was checked with a primer pair (143F/145R) designed by Lo et al. (1996a) and based on a highly conserved region of the 18S rRNA gene sequence of decapods (Kim \& Abele 1990). The thermal cycling programs and reaction conditions for 2-step WSSV diagnostic PCR and DNA quality testing PCR were as described in Lo et al. (1996a).

WSSV screening of Artemia. Primer sets pms 146F1/ R1-F2/R2 and IQ2000 were used for 2-step WSSV diagnostic PCR screening of samples from 3 commercial brands of dry-packed Artemia cysts. These packets of cysts were obtained from the Taiwan Fisheries Research Institute Tung Kang Marine Laboratory, and they were all originally intended for general aquacultural use. The labeling information on Brand A was no longer legible, but its production date was deduced to be no later than 1987 based on other extrinsic information. Brand B was packed in 1997 and Brand C was packed in 1998. Nauplii hatched from Brands B and C Artemia cysts were also subjected to 2-step WSSV PCR screening. The Brand A cysts did not hatch and so no Brand A nauplii could be tested. 
Cysts A, B and C together with cysts from 2 additional samples (D and E, packed in 1999 and obtained from a local commercial source) were subjected to further PCR tests using 3 nested primer sets based on the pms 146 fragment. These were pms 146F1/R4-F3/R5, pms 146F2/R2-F5/R4 and pms 146F4/R2-F5/R4 (Lo et al. 1996a). In addition, 4 other nested PCR primer sets were used. These targeted 2 sequences within the WSSV-rr1 coding region ( 1 by primer set rr1F809/rr1R1426-rr1F863/rr1R1227 and 1 by primer set rr1F1551/rr1R1980-rr1F1726/ rr1R1945), 1 sequence in the WSSV-rr2 coding region (by primer set rr2F704/rr2R1423-rr2F806/ rr2R1008) and 1 sequence in the WSSV-tk-tmk coding region (by primer set tkF310/tkR779-tkF351/ tkR707). The latter primer set yielded an amplicon that included the $t k$-tmk junction region, which is a specific WSSV identification marker (Tsai et al. 2000b). The PCR conditions were the same as described in Lo et al. (1996a) except that the annealing temperature for primer set rr1F809/rr1R1426rr1F863/rr1R1227 was $58^{\circ} \mathrm{C}$, and the one for primer set tkF310/tkR779-tkF351/tkR707 was $52^{\circ} \mathrm{C}$.

The 2-step PCR amplification products yielded by each of the above primer sets were subjected to direct nucleotide sequencing using the respective internal primer pairs. Sequence analysis was performed on an automated ABI Model-377 sequencing apparatus (Applied Biosystems) and carried out by Mission Biotech. DNA samples extracted from nauplii hatched from Brand D and Brand E Artemia cysts were also tested by 2-step WSSV PCR with primer set pms 146F4/R2-F5/R4.

Nucleotide sequence comparison and ARFLP analysis. The nucleotide sequences of the 2-step PCR products amplified from Artemia cysts using WSSV-specific primers were compared with the sequence of Penaeus monodon WSSV 1994 Taiwan isolate (Lo et al. 1996a). Multiple nucleotide sequences were aligned using the programs CLUSTAL_X (Thompson et al. 1997) and GeneDoc (Nicholas et al. 1997). Restriction enzyme digestion sites were analysed with the computer software GeneWorks version 2.5.1 for Macintosh (IntelliGenetics).

On the basis of the sequence analysis data, an ARFLP analysis using either MnlI or BsrI restriction enzyme was developed to distinguish samples from different sources. Two-step PCR products from all brands of Artemia cysts and positive controls were selected for MnlI or BsrI ARFLP analysis. Aliquots of 10 to $15 \mu \mathrm{l}$ of PCR products were digested in a $20 \mu \mathrm{l}$ reaction volume containing the restriction enzyme and an appropriate buffer supplied by the enzyme manufacturer (New England Biolabs). The mixtures were incubated at $37^{\circ} \mathrm{C}(\mathrm{MnlI})$ or $65^{\circ} \mathrm{C}(\mathrm{Bsr} \mathrm{I})$ for $1 \mathrm{~h}$, and
$10 \mu$ aliquots were loaded for electrophoresis. Digested DNA fragments were separated on 5\% Trisborate-EDTA agarose (NuSieve 3:1 agarose, FMC BioProducts), stained with ethidium bromide and examined on a UV transilluminator.

Feeding of nauplii hatched from WSSV-positive Artemia cysts to Penaeus monodon. Uninfected Penaeus monodon larvae from a single WSSV-free (2-step PCR-negative) brooder were used for this challenge test. Special attention was paid to diet. In particular, these larvae were not fed Skeletonema costatum because naturally harvested $S$. costatum is often contaminated with copepods, and Lo et al. (1996b) have shown that copepods are potential reservoir hosts for WSSV. Before the experimental challenge, the larvae were therefore fed exclusively monocultures of the microalgae Isochrysis galbana and Chaetoceros gracilis and a commercial dry diet. As a further precaution before the infectivity trials began, pooled samples from the shrimp larval population were tested for the presence of WSSV, and their 2-step PCR negative status was reconfirmed with both the pms 146F1/R1-F2/R2 and IQ2000 primer sets.

At stage 2 of mysis, 3000 of these Penaeus monodon specimens were divided evenly into three 1501 tanks containing chlorinated, aerated 30 ppt seawater. $P$. monodon in 2 tanks were then fed Artemia nauplii hatched from WSSV PCR-positive cysts Brand B and Brand $C$ while the third tank served as the control. The Artemia cysts were hatched in sterilized 30 ppt seawater at room temperature $\left(25\right.$ to $\left.29^{\circ} \mathrm{C}\right)$ with full aeration for $24 \mathrm{~h}$. After hatching, the nauplii were separated from the empty shells and washed thoroughly with sterilized seawater before being used to feed the $P$. monodon larvae. Twice daily the $P$. monodon larvae were fed 1 meal each of Brand B Artemia nauplii plus commercial dry diet and Brand C Artemia nauplii plus commercial dry diet or 2 meals of dry diet only (control). At $\mathrm{PL}_{15}$, randomly selected specimens were collected and had their heads removed before being placed in pooled samples (10 samples of $100 \mathrm{mg}$ each), which were then independently subjected to both of the 2-step WSSV diagnostic PCR protocols described above ( $2 \mu$ l DNA template per reaction). A positive control DNA template was prepared from the abdominal muscle tissue (100 mg) of a 1-step PCR-positive adult $P$. monodon.

\section{RESULTS}

\section{Two-step WSSV PCR tests of Artemia cysts and nauplii}

Examples of the PCR detection results with the IQ2000 primer set in the first 3 tested brands of 


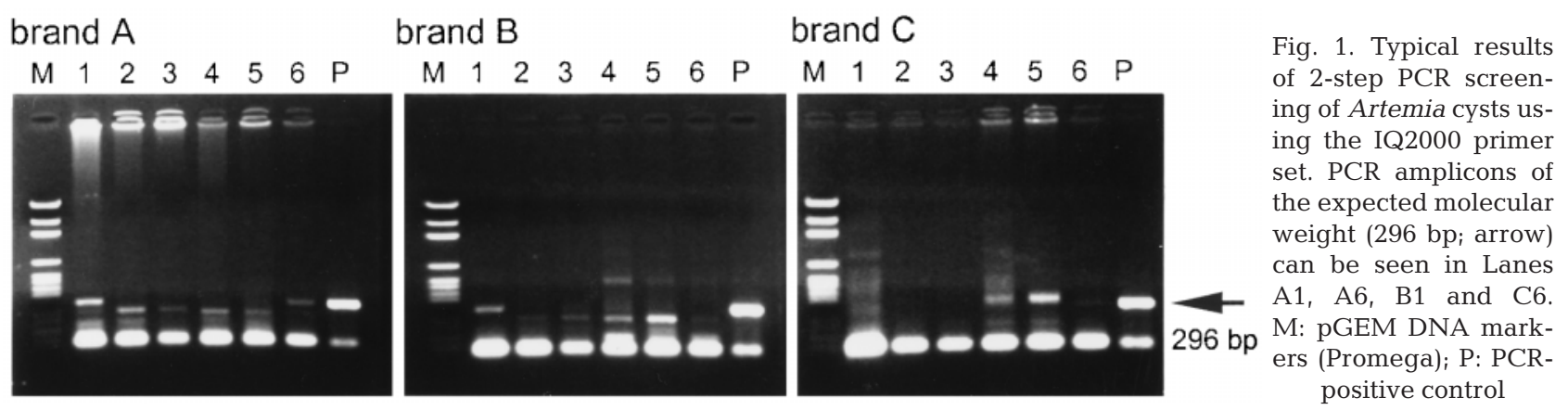

Artemia cysts are shown in Fig. 1. In total, 4 out of 21 tested samples of Brand A gave WSSV positive reactions in the second step of the 2-step PCR while Brands $\mathrm{B}$ and $\mathrm{C}$ each gave 6 positive in 21. However, the primer set pms 146F1/R1-F2/R2 gave negative results for every tested sample of these 3 brands. WSSV negative results were also obtained with all of the hatched Artemia nauplii tested (Brands B and C with the IQ2000 primer set and Brands D and E with the pms 146F4/R2-F5/R4 primer set). All of the above results and results for the other 3 pms 146 primer sets (i.e. pms 146F1/R4-F3/R5, pms 146F2/R2-F5/R4 and pms 146F4/ R2-F5/R4) for all of the 5 brands of Artemia cysts are summarized in Table 1.

\section{Nucleotide sequence comparison and ARFLP analysis of the pms 146 fragments}

Sequences and alignment (with Penaeus monodon WSSV 1994 Taiwan isolate; accession number U50923) of the amplified pms 146F3/R5 and pms 146F5/R4 fragments from each brand of Artemia cyst WSSV are shown in Fig. 2. No sequence differences were found in pms 146F5/R4 amplicons from any brand of Artemia cyst. Single nucleotide variations were found in the pms 146F3/R5 amplicons from
Brand $\mathrm{B}$ and Brand $\mathrm{E}$ (Fig. 2B). A point difference $\left(\mathrm{C}^{296} \rightarrow \mathrm{T}\right)$ in a fragment from Brand B Artemia cysts blocked an MnlI recognition site. In consequence, the MnlI digestion profile of Brand B pms 146F3/R5 amplicons consisted of 3 bands (136, 116 and $18 \mathrm{bp}$ ) rather than the normal 4 (136, 92, 24 and $18 \mathrm{bp}$; Fig. 3A). In Brand $\mathrm{E}$ a $\mathrm{C}^{267} \rightarrow \mathrm{G}$ difference generated a new recognition site for BsrI, Sau96I and HaeIII. In this study BsrI was selected for ARFLP analysis. The Brand E BsrI digestion profile showed a 2 band pattern (216 and $54 \mathrm{bp}$ ) compared with the normal WSSV 270 bp single band pattern (Fig. 3B)

\section{Other WSSV gene fragments amplified from Artemia cysts}

As shown in Table 2, the other 4 WSSV gene-specific primer sets all produced positive results with Brand A Artemia cysts. One of the rr1 primer sets (rr1F809/rr1R1426-rr1F863/rr1R1227) gave no amplicons with cyst Brands $\mathrm{C}, \mathrm{D}$ and $\mathrm{E}$, and the rr2 primer set gave no amplicons with Brands B and D. The other rr1 primer set (rr1F1551/rr1R1980-rr1F1726/ rr1R1945) and the tk-tmk primer set both yielded amplicons with all 5 brands of Artemia cysts tested (Table 2).

Table 1. White spot syndrome virus (WSSV) PCR screening results for Artemia cysts and nauplii using 5 primer sets derived from the WSSV pms 146 genomic fragment. Data are the number of 2-step positive results out of the number of samples tested. - : not tested

\begin{tabular}{|c|c|c|c|c|c|c|}
\hline \multirow{3}{*}{$\begin{array}{l}\text { Cyst data } \\
\text { WSSV pms } 146 \\
\text { primer sets }\end{array}$} & \multirow{3}{*}{$\begin{array}{c}\text { Amplicon } \\
\text { size (bp) } \\
\text { 1st-step / 2nd-step }\end{array}$} & \multicolumn{5}{|c|}{ Brand and year of packaging } \\
\hline & & A & B & C & $\mathrm{D}$ & $\mathrm{E}$ \\
\hline & & before 1987 & 1997 & 1998 & 1999 & 1999 \\
\hline F1/R1-F2/R2 & $1447 / 941$ & $0 / 21$ & $0 / 21$ & $0 / 21$ & - & - \\
\hline IQ2000 & $910 / 296$ & $4 / 21$ & $6 / 21$ & $6 / 21$ & - & - \\
\hline F1/R4-F3/R5 & 839 / 269 & $1 / 15$ & $4 / 15$ & $3 / 15$ & $1 / 15$ & $1 / 15$ \\
\hline F2/R2-F5/R4 & $941 / 237$ & $2 / 15$ & $3 / 15$ & $1 / 15$ & $1 / 15$ & $4 / 15$ \\
\hline F4/R2-F5/R4 & $736 / 237$ & $5 / 15$ & $5 / 15$ & $6 / 15$ & $4 / 15$ & $1 / 15$ \\
\hline \multicolumn{7}{|c|}{ Hatched nauplii data } \\
\hline IQ2000 & & - & $0 / 21$ & $0 / 21$ & - & - \\
\hline F4/R2-F5/R4 & & - & - & - & $0 / 5$ & $0 / 5$ \\
\hline
\end{tabular}




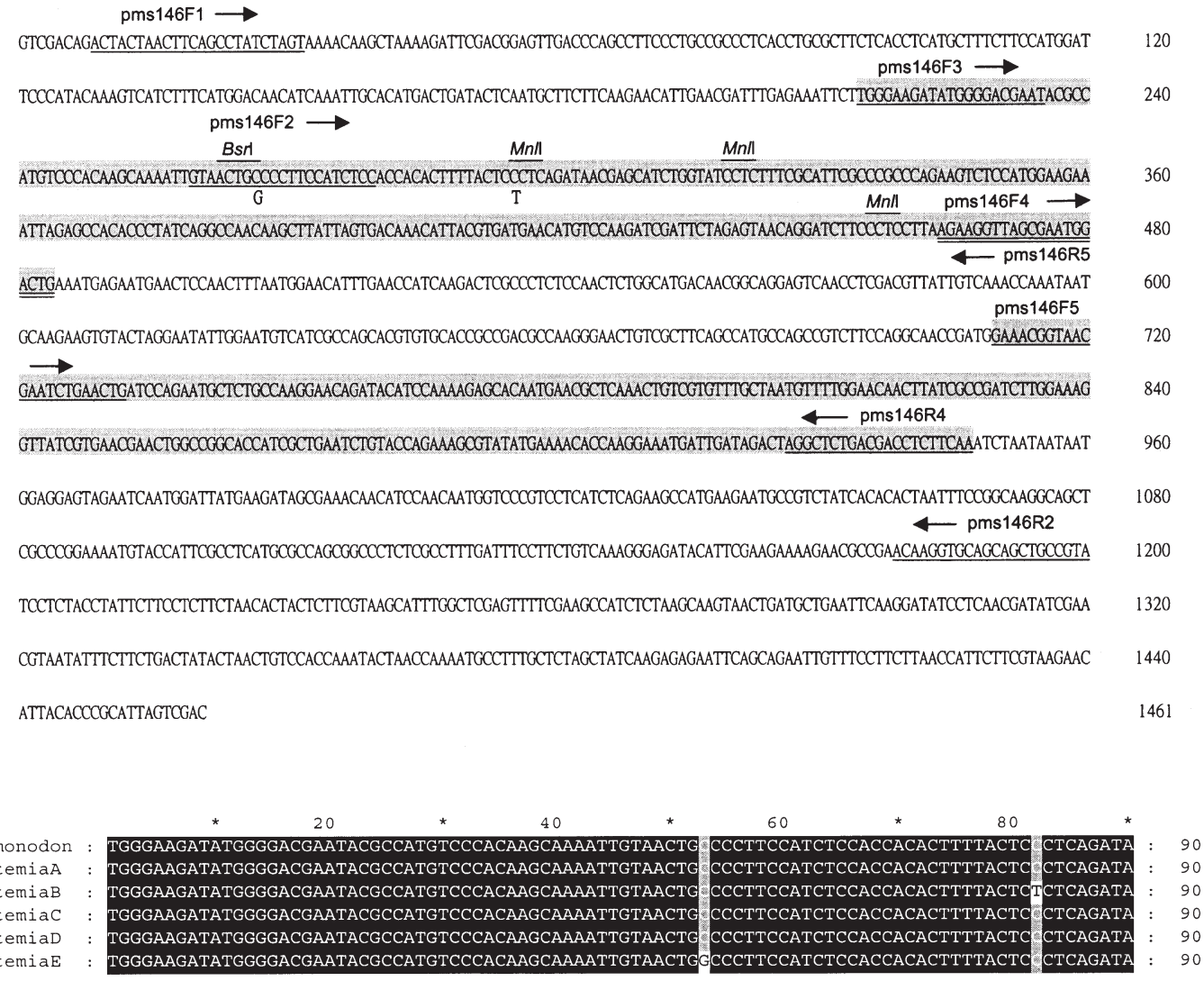

Fig. 2. (A) Full nucleotide sequence of the Penaeus monodon WSSV pms 146 fragment (accession number U50923) showing the PCR primer positions, BsrI and MnlI restriction enzyme recognition sites and the regions in Artemia cyst WSSV that were sequenced (shaded). (B) Nucleotide sequence alignment of pms 146F3/R5 amplicons of Artemia cyst WSSV with P. monodon WSSV 1994 Taiwan isolate. Nucleotide variations were observed in Brand B at location $\mathrm{C}^{296} \rightarrow \mathrm{T}$ and in Brand $\mathrm{E}$ at location $\mathrm{C}^{267} \rightarrow \mathrm{G}$

Nucleotide sequence analysis showed that all of the successfully amplified WSSV gene fragments (Table 2) exactly matched the corresponding sequences in the coding regions of the Penaeus monodon WSSV 1994 Taiwan isolate. For reference, full nucleotide and de- duced amino acid sequences of the $P$. monodon WSSV $r r 1, r r 2$ and tk-tmk coding regions are shown in Fig. 4A, B and C, respectively. Also indicated in Fig. 4 are the PCR primer sets used and the location of the sequenced regions.

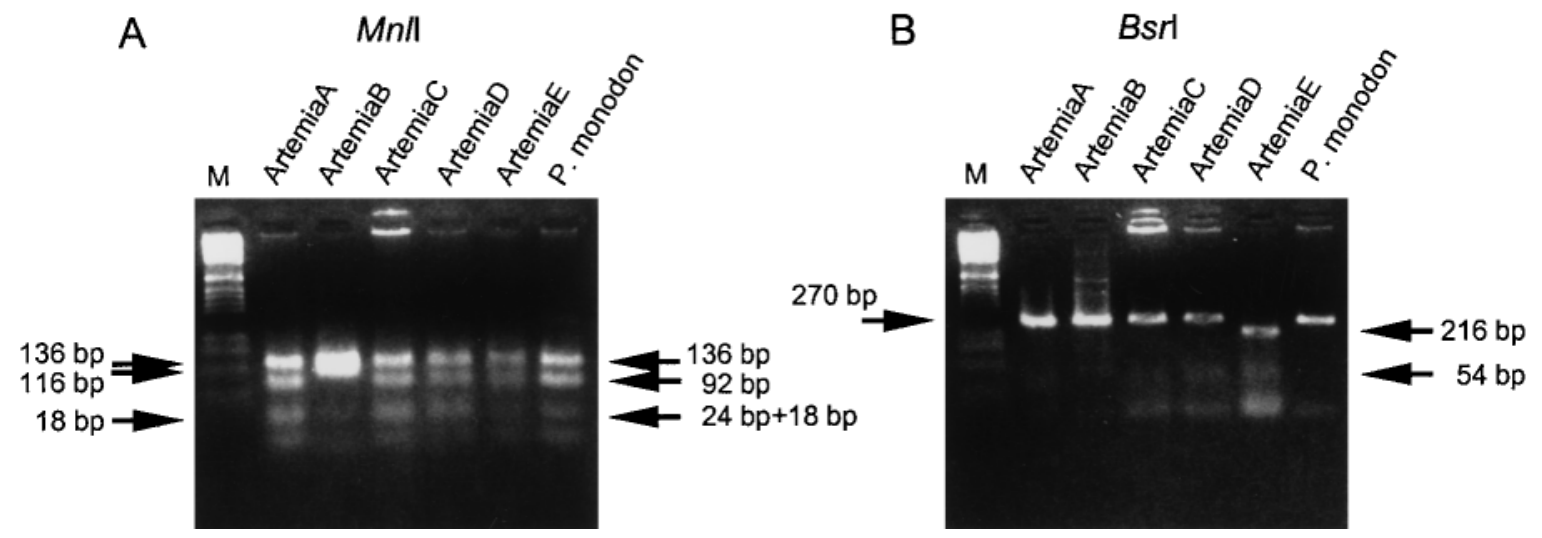

Fig. 3. Restriction profiles of the 270 bp WSSV fragment amplified by pms 146F3/R5. (A) MnlI-digested DNA fragments; (B) BsrIdigested DNA fragments. The Penaeus monodon-positive control DNA fragments were derived from P. monodon WSSV 1994 Taiwan isolate. M: pGEM DNA markers (Promega) 
A ATGGGTTCTAACCAGCAACAATCATTCATCTCAAAGAGGAATGGCACTAAGCAAGAAATTAGTCTTGAAAAGATAATCAAGAGGATTGAAAATGCCTGTCTTCCAGTCAACCAGTATGTG

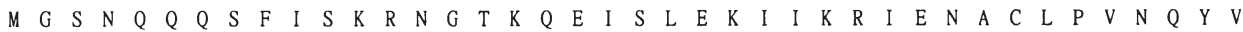
CCCAAGCTTGACAAGAACGCAATTAACCCTCAAGAACTTGCATCTCACATCATGGACCGTCTTCCCGCTACCATCTCCTTCCAAGAAATGGACGATTTTCTGGCCGATTATGCAAAGACA

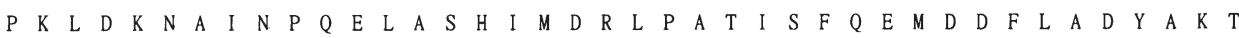
AAAATTGTTGACCACCCTGATTTTGGAAAACTGGCAGGAAGATTCATCTGTTCGAACATCCACAAAAACACCAAAGAGTGGAATAGTTTTAGTGCAACAACTCAGAAATTGAGGCACGCA

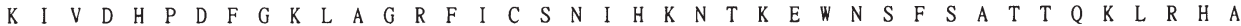
ATCCACCCTGGAACTGGTAAACCAGCATCAGTAGTTAATGATACCTACTATGAAAATGTTATGGCCAATGCTGAAATTCTCGATGCTGTTATCGATTACAAAATGGATTATCTCTTCACC

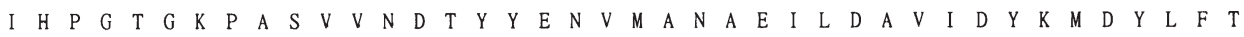
TGTTTCGGACTGAGGACGCTAGAATACTCTTATTTGATCAAGATTGGTTCCCCCACTGATAGAAAGAAGAGAATCTTGGTTGAGCGCCCTCAGGACATGATTATGCGTGTGGCTGTCGGC

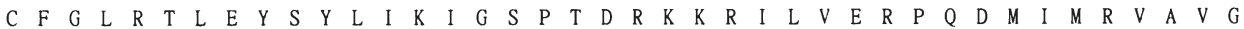

ATTCACGGATCAGACATCAAATCTGTCATTGAAACATATGATCTCATGTCGAGGCACTATTTTACCCATGCTTCCCOCCACACTGTTTAATTGTGGAACAGTCACACCCCAACTTTCCTCG

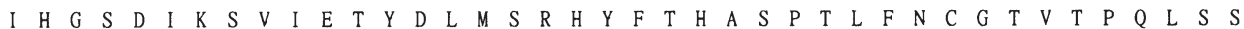
WSSVrr1F809 $\rightarrow$

TGCTTCCTTCTGGGCCTTCAAGATGATAGCATTGAGGGTATTTATGATACTCTTAAGGAGGCGGCAATCATCTCCAAGACTGCTGGAGGACTCGGCATCCACTTTCATGATTTGAGGGCA

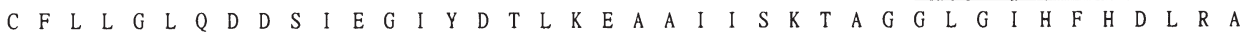
WSSVrr1F863 $\rightarrow$ AAAGGAAGCCCCATTTCGTCATGGAGTGGTACCCACCCTGGTCTCATCGCGTTCCTCCAAATCTTTAACGTCTCTGTGAAAAAGGTTAGCCAGGGAGGAGACAAGAGGAGAGGAGCTCCA

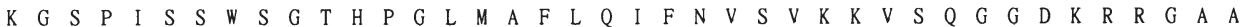

GCCATCTATATTTCTGATTGCCATCTGGACGTGAAGGACTTTATTGACTCCAGAAAGAATGCOCGTAATGAAGATTTGAGGACGAGGGATCTTTTCOCAGCTATCTGGGTATCIGATCTC

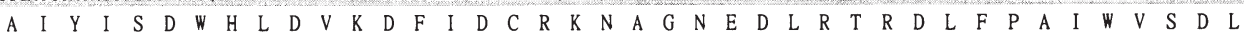
TTCATGGAGAGAGTGAAGCCTGGGAAGAATTGGTCCCTGATGTGCCCCCACGAGTGCCCTTGGCCTTTCCGACGTCCATGGOGAAGAGTTTAAGGCGTTGTACGAGAAATATGAGGCTGAA

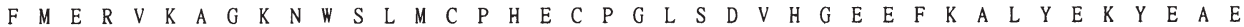
$\longleftarrow$ WSSVrr1R1227

GGCAAAGGTAAAGAGGTGGTGAAGGCACGTGCATTATTCGACCAAATTAATTCTGCACGTATCGAAACTGGAACACCTTATGTGTGCTTTAAGGATACCATCAATAGAAAGTCTAACCAA $\begin{array}{lllllllllllllllllllllllllllllllllllllllll}G & K & G & K & E & V & V & K & A & R & A & L & F & D & Q & I & N & S & A & R & I & E & T & G & T & P & Y & V & C & F & K & D & T & I & N & R & K & S & N & Q\end{array}$ $\leftarrow$ WSSVrr1R1426

GAAAATGTCGGCATCATCAAGTCTTCAAATTTGTGCACTGAAATTGTCCAGTACAGTGATTCGGAGGAAACTGCAGTGTGCAATTTGGCTTCTATCGCAGTCAACAAGTTTGTGAAGTAT

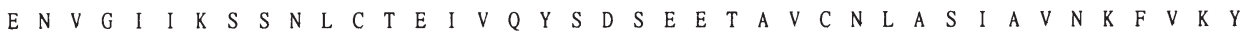
WSSVrr1F1551 TCTCCCATCCCTTCCCTAAGGCCCTATGTTGATTACCGGGAGATGAAGAGGGTTGTAAAAATCATGACCAGAAATCTCGACAAGGTGATTGATGTCAATTTCTATGCAGTTGACAAGACC

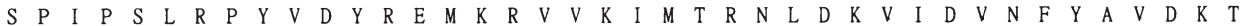
$\rightarrow$ CGCATTTCCAATATGAAAACTAGGCCAATGGGATTGGGTGTGCAGGGACTAGCAGATTTGTTCTTCAAACTCAGAATCCCCTTCGAATCTGAAGAAGCGGCACTAATTAACAAGAGGATT $\begin{array}{lllllllllllllllllllllllllllllllllllllllll}R & I & S & N & M & K & T & R & P & M & G & L & G & V & Q & G & L & A & D & L & F & F & K & L & R & I & P & F & E & S & E & E & A & A & L & I & N & K & R & I\end{array}$ WSSVrr1F1726 $\rightarrow$

TTTGAAACTATATACTATGGTGCCTTGGAAGCTTCATGTGAAATTGCCAAAGAAAAGGGAGAAACATATGAGCTGTTIGAGGGTAGTCCTTTGAGCAAAGGGATTTTCCAATTTGACATG

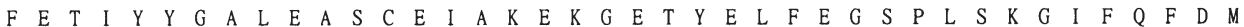

GGGAAGGAAAATATTAAGAATAGGGACATATATTTCAACTCTTTGCCAATTCACGATTGGGACCAATTGAGAAGGGACATTATGAAGTATGGTGTICACAATTCAATGTTTGTTCCTCCC

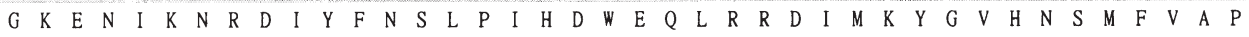
$\longleftarrow$ WSSVrr1R1945 $\longleftarrow$ WSSVrr1R1980 ATGCCTACTGCATCCACTGCACAGATOCTGGCCAACTCTGAATCCTTTGAGCCTTTAACGTCCAACATGTATAATCGTAATGTACTTTCAGGATCATTCCAAGTGGTGAACGAATATGTG

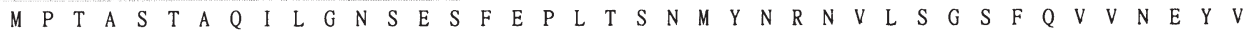

ATTAGAGAGCTCATAAAACTGGGAGAATGGAATTCAGTAACTAAACAGAGGATTATGGCAAGTGGTGGATCTATTCAGACGCTTCCTAATATCCCTAAATCGACCAAGGAACTATTCAAA

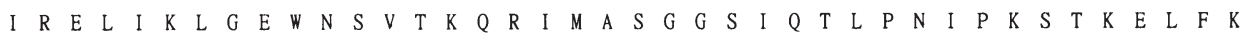
ACTGTATGGGAAATTAATCCTCGTACTACTTTGGACATGGCTATTCAGAGAGGTATGTTTGTTGACCAAGCTCAATCCCTCAACTTGTTTGTGGAAGAACCCGAACTCAGCAAGGTGCGG

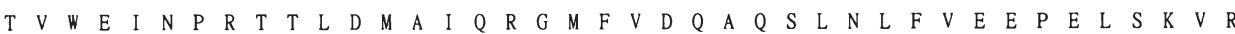

TCAATGACTATGTATGCATGGGAGAAGGGGATCAAGACTCTCTATTATCTACGCACAAAGGGCGCAGCTAGAGCTGTCCAGTTCACAGTCGACAAGAATGTACTCCAAGAAGTCAAGAAG

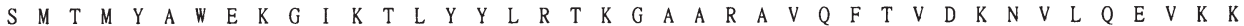
GAAGCTCCCTCTCCTGTTGCAGCTTTTTCTGCTCCTGTCCGAGAAGAAGAAGAGGAGAAGAAGTCCTCTATTGTTGTTCCAGATCCTGCTGCTGCTCTTTTATGTTCTATCAATAACCCT

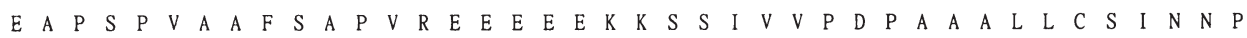
GGTGCTTGTGAAATGTGTTCTTCCTAG G A C E M C S S S *

120

240

360

480 600 720 840

Fig. 4. (Above and facing page). Nucleotide and deduced amino acid sequences of the coding regions of 3 genes of Penaeus monodon WSSV 1994 Taiwan isolate. (A) $r r 1 ;$ (B) $r r 2 ;$ (C) tk-tmk. The positions of the PCR primer sets are shown. Shading is used to indicate the sequenced regions. The boxed sequence in $(C)$ indicates the $t k-t m k$ junction region 
B ATGGAGTCAATCAAACTGTTCACCGTTGCTGGTCTGAATATGGAGCAAGCCAACCAAGTGGCTGAAGAAATCAAGTCAGAATATAAAACCGAGGAGGAAAAGAGGATTGCCCAGGAAGTG

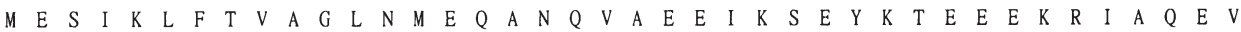

TTTGACAAATTCACCAAAAAACTCATTATGCAAGTAGATACGTCTAAACACTTACTTACAAGAGAAAACCCCAACCGTTTTGTATCCCGCCCCATTGTCCATGAAGATCTCTGGGAAATG

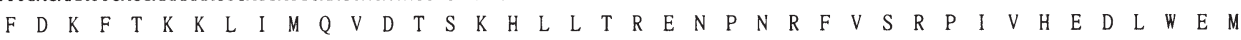

TACAAAAAAGAGGTTGCCTGTTTTTGGACATTGGAAGAGATTGATTTCGAAAGGGATCCTAAAGATTGGGAGAAACTCACTCAAGATGAGAAGGATTTCATTCTCCAGATTCTGGCGTTC

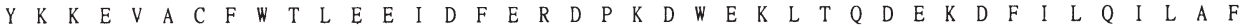
TTTGCATCCTCTGACGGAATTGTAATTGAAAATCTTACAACACGTCTTCGTCAAGTGGCGCAGATTCCAGAAGCGAGGAGTTTCTTTGACTTCCAAGTTGGAATGGAGAGTATTCATGGC

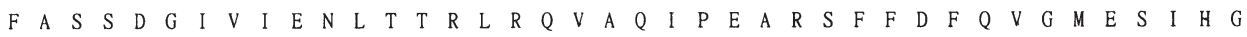
AACGTCTACGGAGAACTGATTGATAGACTGGTGCCCGACGAAAAAGACAAGGCTATCTTGTTTAACGCTGCACAACACTTCCCCGCCATCAAGAAGAAGGAGCAGTGGGCTATTAATTGG $\begin{array}{lllllllllllllllllllllllllllllllllllllllll}N & V & Y & G & E & L & I & D & R & L & V & P & D & E & K & D & K & A & I & L & F & N & A & A & Q & H & F & P & A & I & K & K & K & E & Q & W & A & I & N & W\end{array}$ WSSVrr2F704 $\rightarrow$ ATGCAAAGCAATAACGATTTGGCGGAACTAATTGTTGCCTTTGCTGCAGTTGAAGGAATCTTCTTTAGTGGTGCATTCGCATCCATTTTCTGGATCAAGAACAGGGGTATTTTGCCTGGT

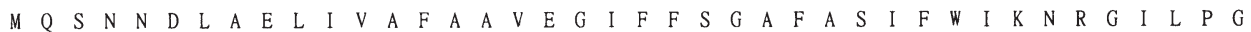
WSSVrr2F806 $\longrightarrow$

CTCACCTCCTCCAATGAGTTCATTTCTAGGGACGAAGGTCTTCATCGCGACTTTGCATGCATGCTGTTGAAAAAGGGTTTTGTTGATACCCCATCAAGAGAAAGGATTCTTGAAATTGTC

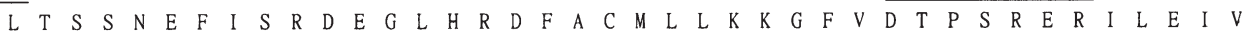
ACTGAAGCCGTCCGAATTGAACAAGAATTTCTCACAGTTTCCCTGCCTGTTAAATTAGTGGGAATGAACTGCAAGTTGATGAGCCAGTACATTGAATTIGTGGCAGATAAACTATTGGTT

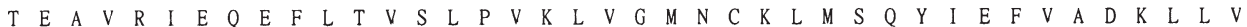
$\longleftarrow$ WSSVrr2R1008

GAAATGGGACTAGAAAAGCACTATAATGTTACCAACCCCTTCCCATTCATGGACAATATTTCCCTCGAGAATAAGACCAACTTTTTTGAAAAGAGAGTCGCCGAGTATCAACGTGCCCAG

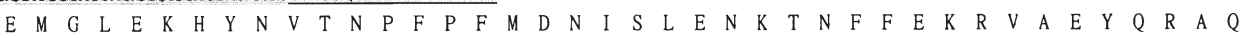
GTCATGGCTTCTATCAATAAGATCAAGAAGGACCAACAAACCCAAGAAACTGGTTCTCCTCTCCCAATTCTGACTGCACCTCCTCCAGTCTCTTCCTCATCATCCGAACAAGAAGATGTT

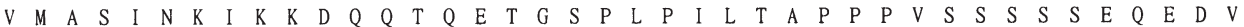

GAAGACGGCGTCGGGGACTACATCAGTTATGACGATTTTTAGTTCCACTATTGTGTCAATAGGTTGTGTATTGTATTATTATTGTTATAATATTTTTAAAAAATAAATGTTCTATAAGAC

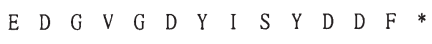

C ATGGCTGGTCGTGTAGAGCTCGTCACTGGACCCATGTTTGCGGGCAAGTCTACCTACCTGAAAAACATATACCAACAAGAAAATGGAGGCAATAAACATTGCCTGTTTGTCAAACACTCC

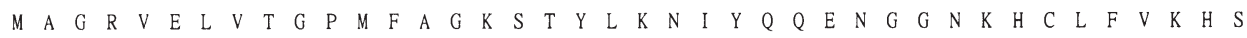

CTAGAAACTAGGTACGGTTGTGGAACTGGAACAATAGTCACTCATGCCGGAGAAGTGATTGAAGGTTGTACTACAGTTTCTTCTATCAAGGAACTAATCAGTGTGTTACCAGAAGTTGTO

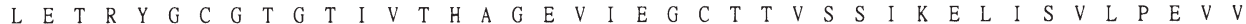
WSSVtkF310 $\longrightarrow$ WSSVtkF351 GATGTGATTCTCATTGACGAAGGGCAATTCTTCACGGATTTGGTGCTAGTCAATAGACTGGCTGACAAGGGGAAAAGGATTGTGATTGCAGCACTTGATGGAACTTCTGACCAGCAAATO $\begin{array}{lllllllllllllllllllllllllllllllllllllllll}D & V & I & L & I & D & E & G & Q & F & F & T & D & L & V & L & V & N & R & L & A & D & K & G & K & R & I & V & I & A & A & L & D & G & T & S & D & Q & Q & M\end{array}$

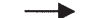
$\longrightarrow$ ITCAGTCCTATTCATAAGCTATTGCCTTATACAAATTCCATTGTTAAGCTAGCATCTAAATGTATGATTTGTAAAATTGATACCAAAGAAGCTCCTTTTACTGTAAGGTTTCGTAATGAC $\begin{array}{llllllllllllllllllllllllllllllllllllllllll}F & S & P & I & H & K & L & L & P & Y & T & N & S & I & V & K & L & A & S & K & C & M & I & C & K & I & D & T & K & E & A & P & F & T & V & R & F & G & N & D\end{array}$ AATGATAATAATGTTATATGTGTAGGAGGAGCTGAAATGTACGCTGCTCCCTGCCGGGACTGTTACAAAAAAATTAACAAGAAAAAGAACAAGGGGAAACTTGTTGTACTTGAAGGAGGT $\begin{array}{lllllllllllllllllllllllllllllllllllllllll}N & D & N & N & V & I & C & V & G & G & A & E & M & Y & A & A & A & C & R & D & C & Y & K & K & I & N & K & K & K & N & K & G & K & L & V & V & L & E & G & G\end{array}$ $\longleftarrow$ WSSVtkR707 GACAGGTGCGGTAAGAGTACCCAAGCCAAACTCTTGTTGACCAATAAAAACTCGCCTCTTTATGGAGGAGAATACATGTGCTTTCCCGACAGGAGCAGCCATACGGGTAAACTCATCAAT $\begin{array}{lllllllllllllllllllllllllllllllllllllllll}D & R & C & G & K & S & T & Q & A & K & L & L & L & T & N & K & N & S & P & L & Y & G & G & E & Y & M & C & F & P & D & R & S & S & H & T & G & K & L & I & N\end{array}$ WSSVtkR779 GATTATTTAACTAAGAAAATTGAACTAGATGATCATGCAGCTCACTTGTTATTTTCTGCAAATAGATGGGAAGTTTGTAGTAAAATTAAGCAGTTGTTAGACGATGGAATCCATGTTGTO

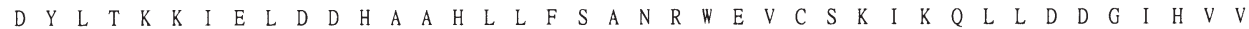
ATGGATAGATATTACTACTCGGGGATTGTTTTCTCTTTAGCTAGAGGAGTGGATACCGTTGAGTGGTGCTCTGCTAGCGATGAGGGACTTCCTCAGCCCGATCTTGTATTGTTGATGCTT

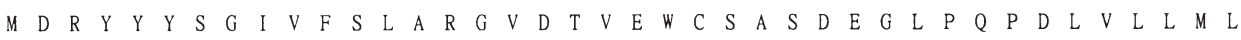

TTAGATGTTGAAAAGTGTTCAAATAGGGATACTTTTGGTGTCGAAAGATTTGAGACAAATTCCATTCAAGAACGTGCTAGAGCCCTATTCCTAGACCTCGCAAATAAGGACGAAAAGAAT

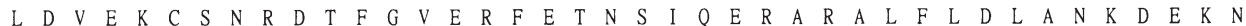


Table 2. Recognition of specific WSSV rr1, rr2 and tk-tmk gene fragments in Artemia cyst WSSV. Two-step PCR was performed on DNA samples from WSSV-positive Artemia cysts using primer sets for 4r WSSV gene fragments. Pooled samples from each brand were tested up to 5 times with each primer set. Data show the number of times a sample was tested (n) before a PCR-positive signal was seen $(1 / \mathrm{n})$ or indicate that a sample was tested 5 times without a positive reaction $(0 / 5)$

\begin{tabular}{|c|c|c|c|c|c|}
\hline \multirow{2}{*}{ WSSV primer sets } & \multicolumn{5}{|c|}{ Brand and year of packaging } \\
\hline & $\begin{array}{c}\text { A } \\
\text { efore } 1987\end{array}$ & $\begin{array}{c}\mathrm{B} \\
1997\end{array}$ & $\begin{array}{c}\mathrm{C} \\
1998\end{array}$ & $\begin{array}{c}\mathrm{D} \\
1999\end{array}$ & $\begin{array}{c}E \\
1999\end{array}$ \\
\hline $\begin{array}{l}\text { rr1F809/rr1R1426- } \\
\text { rr1F863/rr1R1227 }\end{array}$ & $1 / 5$ & $1 / 3$ & $0 / 5$ & $0 / 5$ & $0 / 5$ \\
\hline $\begin{array}{l}\text { rr1F1551/rr1R1980- } \\
\text { rr1F1726/rr1R1945 }\end{array}$ & $-1 / 4$ & $1 / 1$ & $1 / 4$ & $1 / 3$ & $1 / 1$ \\
\hline $\begin{array}{l}\text { rr2F704/rr2R1423- } \\
\text { rr2F806/rr2R1008 }\end{array}$ & $1 / 5$ & $0 / 5$ & $1 / 5$ & $0 / 5$ & $1 / 5$ \\
\hline $\begin{array}{l}\text { tkF310/tkR779- } \\
\text { tkF351/tkR707 }\end{array}$ & $1 / 5$ & $1 / 4$ & $1 / 5$ & $1 / 4$ & $1 / 5$ \\
\hline
\end{tabular}

\section{Results from feeding Penaeus monodon with nauplii hatched from WSSV-positive cysts}

All tested Penaeus monodon PL from the 2 test groups and the control group were 2-step WSSV PCR-negative with the pms 146F1/R1-F2/R2 and the IQ2000 primer sets (data not shown). The survival rates from stage 2 mysis to $\mathrm{PL}_{15}$ were about $50 \%$ in both of the groups fed Artemia while that in the control group (commercial diet only) was approximately $20 \%$. Growth rates were also very similar in the 2 Artemia-fed groups and slightly lower in the group fed commercial diet only.

\section{DISCUSSION}

The well-known importance of Artemia in the larval shrimp diet was illustrated again in the present study where Penaeus monodon PL fed a dry commercial diet alone had a lower growth rate and survival than the 2 groups fed nauplii. We attribute this result both to the inadequacies of the artificial diet used and to the cannibalistic behavior of the shrimp larvae in the test.

Although WSSV-specific PCR tests gave positive results for all 5 of the tested brands of Artemia cysts, all of the tested nauplii derived from those cysts and all of the tested shrimp fed those nauplii gave negative PCR tests for WSSV. There are several possible explanations for these results. The simplest is that the cysts were externally contaminated with WSSV particles, WSSV DNA or WSSV DNA fragments of unknown derivation and that this was removed or diluted to undetectable levels when the hatched nauplii were washed with clean, sterile sea water before being fed to the shrimp. In support of this contention was that the template DNA was present in only very low quantities as evidenced by the facts that nested PCR was required for detection and that only 58 (17\%) of 351 samples tested gave WSSV PCR-positive results.

On the other hand, it was also possible that the WSSV template DNA was present within the cysts and that Artemia might be susceptible to WSSV infection. To accept this very unwelcome prospect it would be necessary to explain the negative WSSV PCR results with the hatched nauplii and the shrimp larvae that ate them. For the hatched nauplii, this is difficult, since any internal viral DNA should have survived washing, however vigorous, and at least some of the samples tested should have given positive results. On the other hand, any virus within the cyst membrane but outside the naupliar membrane might have been washed away. For subsequent disease transfer from the nauplii to the shrimp, it is likely that nauplii actually infected with viable WSSV would have been required at the threshold levels needed to cause shrimp infections. As already mentioned, the cysts contained only very low levels of target template and it is possible that even viable, internal WSSV at such low concentrations would not have been able to infect the shrimp larvae. Thus, despite negative WSSV PCR results for the nauplii and the larvae to which they were fed, we cannot dismiss the possibility that low-level undetectable infections might have occurred in our tests. Specific Artemia infection trials would be necessary, accompanied by histological examination and in situ DNA hybridization tests in addition to PCR tests. Finally, small detected sequence variations from the originally described WSSV of shrimp (see discussion below) open the possibility for the presence of a new variety of WSSV that may not infect shrimp.

Since the IQ2000 primer set targets a smaller amplicon, the different results from the pms 146 primer pair (F1/R1-F2/R2) with cyst Brands A, B and C in Table 1 and Fig. 1 may simply be a consequence of IQ2000's greater sensitivity. This is because primers for smaller amplicons are generally more sensitive (Lo et al. 1996b). However, since both primer sets were designed based on the sequence of the Penaeus monodon WSSV 1994 Taiwan isolate pms 146 fragment, it is also possible that the differences arose from sequence differences in the target DNA. Indeed, 2 point mutations were found in this region (Fig. 2, Brands B and E). More significantly, there were also possible sequence differences in the coding regions of the rr1 and rr2 genes since the rr2 primer set and 1 of the rr1 primer sets failed to detect their respective target fragments in some of the cyst brands tested (Table 2). However, other explanations of the results shown in Table 2 are also possible. For example, the quantity of target DNA 
in some of the samples might simply have been below the detection threshold of the PCR assay, although the $t k$-tmk primer set did produce a positive response in all 5 cyst brands. On the other hand, the potential genetic variation found also suggests that the positive PCR results and negative bioassay results might be explained by the presence of a type or variety of WSSV DNA that is non-infectious for Artemia or shrimp and derived from other arthropods or crustaceans in the Artemia environment.

WSSV is the only organism known to have its $t k$ and tmk genes fused together to form a chimeric $t k$-tmk gene. This gene is therefore likely to be a good marker for identifying WSSV-related viruses (Tsai et al. 2000b) and here the $t k$-tmk primer set that targeted the $t k$-tmk junction region gave positive results for all 5 brands of cysts tested (Table 2). Furthermore, sequence comparisons between the corresponding coding regions of the successfully amplified $r r 1, r r 2$ and $t k$-tmk gene fragments (Table 2) showed complete identity with the Penaeus monodon WSSV 1994 Taiwan isolate (for sequences, see the shaded regions in Fig. 4).

We conclude that WSSV PCR-positive results can sometimes be obtained using several WSSV-specific primer pairs with batches of Artemia cysts and that the PCR amplicons obtained have very high sequence homology to those of WSSV from shrimp. Because of the nature of PCR testing, we cannot say whether such tests indicate the presence of a viable virus, inactivated virus or simply complete or fragmented viral DNA. Indeed, it is very unlikely that the cyst sample dating from 1987 (13 yr old) contained viable WSSV. Nor can we conclude whether the PCR target template was originally present externally or within the cysts. Fortunately, the PCR tests on hatched Artemia nauplii were negative for WSSV, as were the tests on PL that were fed those nauplii. We have also been informed by D. V. Lightner (pers. comm.) that because of import restrictions, Artemia cysts and adult Artemia biomass have been undergoing testing for more than a year for WSSV in his laboratory and those of several others in the USA and Mexico using various primer sets, and that no WSSV-like DNA has been detected. Again, the fact that the adult Artemia where negative in all those tests and in ours supports the argument that our positive PCR tests resulted from contamination of the cysts by something in the Artemia environment. This possibility for external contamination highlights the importance of the usual hatchery practice of washing Artemia cysts with chlorinated water before hatching. On the other hand, the results still leave open a small possibility that Artemia can be infected with WSSV, and this possibility must be vigorously tested, given the enormous impact that a positive outcome would have on the shrimp aquaculture industry.
Acknowledgements. This work was supported by the National Science Council under grant No. NSC 89-2311-B002-078 and the Council of Agriculture under grant no. 90 AS-2.1.1-FA-FI(I2). We are indebted to Dr. Mao-Sen Su and Dr. Huei-Meei Su of Tung Kang Marine Laboratory, Taiwan Fisheries Research Institute, for providing the Artemia cysts, mono-cultured microalgae and culture facilities. We thank Mr. Paul Barlow for his helpful criticism of the manuscript.

\section{LITERATURE CITED}

Austin B, Allen DA (1982) Microbiology of laboratory-hatched brine shrimp (Artemia). Aquaculture 26:369-383

Bardach JE, Ryther JH, McLarney WO (1972) Aquaculture: the farming and husbandry of fresh water and marine organisms. Wiley-Interscience, New York

Beard TW, Wickins JF, Arstein DR (1977) The breeding and growth of Penaeus merguiensis de Man in laboratory recirculation systems. Aquaculture 10:275-289

Chou HY, Huang CY, Wang CH, Chiang HC, Lo CF (1995) Pathogenicity of a baculovirus infection causing white spot syndrome in cultured penaeid shrimp in Taiwan. Dis Aquat Org 23:165-173

Cook ML, Murphy MA (1969) The culture of larval penaeid shrimp. Trans Fish Soc 98:751-754

Criel G (1980) Morphology of the female genital apparatus of Artemia: a review. In: Persoone G, Sorgeloos P, Roels OA, Jasper E (eds) The brine shrimp Artemia, Vol 1. Morphology, genetics, radiobiology, toxicology. Universa Press, Wetteren, p 75-86

Emmerson WD (1984) Predation and energetics of Penaeus indicus (Decapoda: Penaeidae) larvae feeding on Brachionus plicatilis and Artemia nauplii. Aquaculture 38:201-209

Flegel TW (1997) Special topic review: major viral diseases of the black tiger prawn (Penaeus monodon) in Thailand. World J Microbiol Biotechnol 13:433-442

Kim W, Abele LG (1990) Molecular phylogeny of selected decapod crustaceans based on 18s rRNA nucleotide sequences. J Crustac Biol 10:1-13

Kim J, Kenneth CM, Ronald WH (1996) Adult Artemia as food for first feeding coho salmon (Oncorhynchus kisutch). Aquaculture 144:217-226

Lo CF, Kou GH (1998) Virus associated white spot syndrome of shrimp in Taiwan: a review. Fish Pathol 33:365-371

Lo CF, Leu JH, Ho CH, Chen CH and 8 others (1996a) Detection of baculovirus associated with white spot syndrome (WSBV) in penaeid shrimps using polymerase chain reaction. Dis Aquat Org 25:133-141

Lo CF, Ho CH, Peng SE, Chen CH and 7 others (1996b) White spot syndrome baculovirus (WSBV) detected in cultured and captured shrimp, crabs and other arthropods. Dis Aquat Org 27:215-225

Lo CF, Chang YS, Chin CT, Kou GH (1998) PCR monitoring cultured shrimp for white spot syndrome virus (WSSV) in growout ponds. In: Flegel TW (ed) Advances in shrimp biotechnology. National Center for Genetic Engineering and Biotechnology, Bangkok, p 281-286

Mortensen SH, Evensen O, Rodseth OM, Hjeltness BK (1993) The relevance of infectious pancreatic necrosis virus (IPNV) in farmed Norwegian turbot (Scophthalmus maximus). Aquaculture 115:243-252

Naessens E, Lavens P, Gomez L, Browdy CL, McGovernHopkins K, Spencer AW, Kawahigashi D, Sorgeloos P (1997) Maturation performance of Penaeus vannamei co-fed Artemia biomass preparations. Aquaculture 155: 87-101 
Nakano H, Koube H, Umezaea S, Momoyama K, Hiraoka M, Inouye K, Oseko N (1994) Mass mortalities of cultured kuruma shrimp, Penaeus japonicus, in Japan in 1993: epizootiological survey and infection trials. Fish Pathol 29:135-139

Nicholas KB, Nicholas HB Jr, Deerfield DW II (1997) GeneDoc: analysis and visualization of genetic variation. EMBNEW NEWS 4:14

Overstreet RM, Stuck KC, Krol RA, Hawkins WE (1988) Experimental infection with Baculovirus penaei in white shrimp Penaeus vannamei (Crustacea: Decapoda) as a bioassay. J World Aquacult Soc 19:175-187

Sorgeloos P (1980) The use of the brine shrimp Artemia in aquaculture. In: Persoone G, Sorgeloos P, Roels OA, Jasper E (eds) The brine shrimp Artemia, Vol 3. Ecology, culturing, use in aquaculture. Universa Press, Wetteren, p 25-46

Sorgeloos P, Persoone G (1975) Technological improvements for the cultivation of invertebrates as food for fishes and crustaceans II. Hatching and culturing of the brine shrimp, Artemia salina L. Aquaculture 26:369-383

Spann KM, Lester RJG (1997) Special topic review: viral diseases of penaeid shrimp with particular reference to four viruses recently found in shrimp from Queensland.

Editorial responsibility: Timothy Flegel,

Bangkok, Thailand
World J Microbiol Biotechnol 13:419-426

Takahashi Y, Itami T, Kondom M, Maeda M, Fujii R, Tomonaga S, Supamattaya K, Boonyaratpalin S (1994) Electron microscopic evidence of bacilliform virus infection in Kuruma shrimp (Penaeus japonicus). Fish Pathol 29: $121-125$

Thompson JD, Gibson TJ, Plewniak F, Jeanmougin F, Higgins DG (1997) The CLUSTAL_X windows interface: flexible strategies for multiple sequence alignment aided by quality analysis tools. Nucleic Acids Res 25:4876-4882

Tsai MF, Lo CF, van Hulten MCW, Tzeng HF and 6 others (2000a) Transcriptional analysis of the ribonucleotide reductase genes of shrimp white spot syndrome virus. Virology 277:92-99

Tsai MF, Yu HT, Tzeng HF, Tzeng HF and 9 others (2000b) Identification and characterization of a shrimp white spot syndrome virus (WSSV) gene that encodes a novel chimeric polypeptide of cellular-type thymidine kinase and thmidylate kinase. Virology 277:100-110

van Hulten MCW, Tsai MF, Schipper CA, Lo CF, Kou GH, Vlak JM (2000) Analysis of a genomic segment of white spot syndrome virus of shrimp containing ribonucleotide reductase genes and repeat regions. J Gen Virol 81: $307-316$

Submitted: March 3, 1999; Accepted: July 20, 2001

Proofs received from author(s): March 26, 2002 